\title{
ALGORITHMIC EXPLORATION OF DOMINANT TERMS AROUND HAIRPIN VORTICES GENERATED DURING BOUNDARY-LAYER TRANSITION UNDER FREE-STREAM TURBULENCE
}

\author{
KAZUO MATSUURA \\ Graduate School of Science and Engineering, Ehime University, Japan
}

\begin{abstract}
A new method that evaluates dominant local dynamics by skeletonization, mathematical term decomposition and the re-combination of a reduced number of dominant terms around the skeleton points is proposed to clarify the dynamics of hairpin vortices generated during the boundary-layer transition under free-stream turbulence (FST). The development of the method is based on the results of direct numerical simulations conducted for the laminar-turbulent transition on a flat plate with FST intensities of $0-6 \%$ and a free-stream Mach number of 0.5 . Regarding the skeletonization, a new algorithm for extracting the interior points of vortex structures represented by enclosed iso-surfaces is developed. To identify the dominant terms, governing equations are decomposed into non-further-decomposable (NFD) terms. The proposed method is also extended to time series flow field data to reveal the variation of the combination set of dominant NFD terms during the evolution of vortex structures. The present method enables the automatic finding and categorization of the variations of the sets of dominant terms that govern local dynamics during the evolution of hairpin vortices.

Keywords: boundary layer, direct numerical simulation, hairpin vortex, laminar-turbulent transition, stability, turbulence
\end{abstract}

\section{INTRODUCTION}

Hairpin vortices are considered to be elementary building blocks of turbulence near a solid wall [1]. Since the work of Theodorsen [2], there have been many studies on hairpin/horseshoe vortices, and the generation of hairpin vortices has been discussed in numerous reports [3-8]. Among previous studies, Moin et al. [9] numerically examined the deformation of a hairpin-shaped vortex filament under self-induction and in the presence of shear using the Biot-Savart law. Hon and Walker [10] proposed a numerical method based on a Lagrangian vortex method that allows accurate computations of the trajectory of a three-dimensional vortex having a small core radius. Using this method, they clarified that a two-dimensional vortex containing small three-dimensional disturbances distorts into a complex shape, with subsidiary hairpin vortices forming outboard of the original hairpin vortex. Singer et al. [11] computationally studied the formation and growth of a hairpin vortex in a flat-plate boundary layer and its later development into a young turbulent spot. By direct numerical simulation (DNS), Zhou et al. [12,13] studied the evolution of a symmetric pair of quasi-streamwise vortex structures extracted from the two-point correlation tensor of turbulent channel flow data by a linear stochastic estimation procedure. They observed that sufficiently strong hairpin vortices generate a hierarchy of secondary hairpin vortices, and the mechanism of their creation closely resembles the formation of the primary hairpin vortex. Using a compressible DNS, Liu et al. [14] studied the nonlinear stages of laminar-turbulent transition. They discussed the coherent vortex structure appearing in the late stages of transition and the formation mechanism of single vortex rings, multiple vortex rings, and small length scales. Cohen et al. [15] proposed a model consisting of minimal flow elements that evolves into packets of hairpins. The three components of the model are simple shear, a counter-rotating vortex pair 
having finite streamwise vorticity magnitude, and a two-dimensional wavy (in the streamwise direction) spanwise vortex sheet. Eitel-Amor et al. [16] studied the characteristics of hairpin vortices in turbulent boundary layers using parallel and spatially developing simulations. They found that secondary hairpins are only created shortly after initialization, with all rotational structures decaying at later times. Kim et al. [17] studied the effects of background noise on the generation of coherent packets of hairpin vortices. They showed that the hairpins become asymmetric, leading to more complicated packet structures than are observed in the symmetric hairpin vortex train of the flow with a clean background.

Although there have been many studies on hairpin vortices, investigations into the effects of free-stream turbulence (FST) on the evolution of hairpin vortices are relatively scarce. Generally, hairpin vortices extend to various heights from a wall and widths in the spanwise direction. FST makes flow fields more disorganized and difficult to understand compared with flow fields without FST. A new method that can analyse vortex dynamics even under the influence of FST is needed to understand the effects. To the best of my knowledge, important mathematical terms that describe the behaviours of hairpin vortices have not been systematically clarified.

On the other hand, numerous methods have been proposed for vortex visualization $[18,19]$. Among these techniques, one of the most compact methods to represent the feature of a vortex tube is the vortex core representation, or skeleton method. To name a few, Sujudi and Haimes [20] proposed an algorithm for identifying the core of a swirling flow in 3-D discretized vector fields based on the critical point theory. The core is found as the centre of the velocity fields where the rate-of-deformation tensor has one real and a pair of complex conjugate eigenvalues. Kida and Miura [21] proposed the sectional-swirl-and-pressure-minimum scheme, in which individual vortices are visualized by its skeleton representation. Peikert and Roth [22] proposed the "parallel vectors" operator as a basic tool for vector field visualization, and demonstrated various techniques for computing feature lines, such as the vortex core and extremal curves, from field data using this operator in a unified manner. Meanwhile, other published tracing-oriented methods view the problem as integrating an ordinary differential equation. Gelder and Pang [23] proposed a method called "PVsolve" based on a root finding technique. Schafhitzel et al. [24] proposed a vortex core line extraction method based on the $\lambda_{2}$ vortex region criterion. Liu et al. [25] and Gao et al. [26] proposed, by introducing a newly defined tensor "Liutex," a series of third-generation vortex identification methods that can give improved vortex structures and vortex core lines which are unique and thresholdfree. However, these methods are not necessarily combined with the exploration of mathematical terms that describe the behaviours of hairpin vortices.

In this study, a new method that can evaluate dominant local dynamics by skeletonization, mathematical term decomposition, and the re-combination of a reduced number of dominant terms around the skeleton points is proposed to clarify the dynamics of hairpin vortices generated during boundary-layer transition under the influence of FST. In Section 2, computational methods employed in this study are described. In Section 3, computational cases are explained. Section 4 introduces the newly proposed method. Section 5 discusses the effects of FST on hairpin vortex evolution, the point cloud representation of vortices, skeletonization, the selection of the sets of important terms for the system dynamics, and the variation of the sets associated with the time evolution of hairpin vortices. In Section 6, conclusions are presented. In Appendix A, the continuity equation, the compressible Navier-Stokes equations, the energy equation, and the compressible vorticity equations are decomposed into the non-further-decomposable (NFD) terms used in this study. 


\section{COMPUTATIONAL METHODS}

The governing equations are the unsteady three-dimensional fully compressible NavierStokes equations written in general coordinates for body-fitted mesh geometries. The system of equations is closed by the perfect gas law. A constant Prandtl number of $\operatorname{Pr}=0.72$ is assumed. The equations are solved using a sixth-order finite-difference method. Timedependent solutions to the governing equations are obtained using the third-order explicit Runge-Kutta scheme. The present numerical method has been extensively validated for the prediction of transitional and turbulent subsonic flows [27,28].

Regarding the boundary condition, inflow profiles are specified at the inlet. Depending on the computational case, disturbances are added to a base inflow profile. The base inflow profile is obtained by solving the boundary layer equation [29]. At the outflow and upper boundaries, non-reflecting boundary conditions with a mean static pressure of $p_{\infty}=101,325 \mathrm{~Pa}$ are imposed. At the wall, the non-slip, isothermal wall condition $T_{\infty}=273.15 \mathrm{~K}$ is imposed. Periodicity is imposed in the spanwise direction. The computational domain is a rectangular region with dimensions $416.31 \delta_{\text {in }}, 80.0 \delta_{\text {in }}$ and $22.0 \delta_{\text {in }}$ in the $x$ (streamwise), $y$ (wall-normal) and $z$ (spanwise) directions, respectively, for Cases $\mathrm{A}$ and $\mathrm{C}-\mathrm{D}$, and $318.0 \delta_{\text {in }}, 80.0 \delta_{\text {in }}$ and $22.0 \delta_{\text {in }}$ for Case B. Here, $\delta_{\text {in }}$ is the displacement thickness at a virtual point $x=300.79 \delta_{\text {in }}$. The Reynolds number based on the free-stream quantity and the length $\delta_{\text {in }}$ is 1,000 . The inlet position of the computational domain for Cases A and C-D is $x=300.79 \delta_{\text {in }}$, and that for Case B is $5 \delta_{\text {in }}$. The number of mesh points is basically $1,001 \times 241 \times 128$ in the $x, y$ and $z$ directions, respectively, for Cases A and C-D, and $765 \times 241 \times 128$ for Case B.

\section{COMPUTATIONAL CASES}

Four cases (A-D) are considered in this study. The scenario for each case is summarized in Table 1. Cases A and B are reference examples. Case A has no FST, and the transition of the K-regime is reproduced. Case B has FST with a high intensity, Tu $=6 \%$, and produces a bypass transition. In Cases C-D, the evolution of hairpin vortices is influenced by FST. The FST has an intensity of $0.5 \%$ in Case C, $1 \%$ in Case D. The mean free-stream Mach number is 0.5 in all cases. In Case A, disturbances comprising a two-dimensional Tollmien-Schlichting wave and a pair of oblique waves are superimposed on the Blasius solution [14]. In Cases $\mathrm{B}-\mathrm{D}, \mathrm{FST}$ is superimposed on the free-stream region of the velocity profile. In Cases C-D, the initial conditions are the resultant instantaneous flow field of Case A. At the inlet, the sinuous disturbances from Case A and isotropic FST are added to the inflow laminar boundary layer profile.

In the present study, FST is obtained from an instantaneous flow field of isotropic turbulence that had been computed separately. Velocity fluctuations in planes with a constant

Table 1: Computational cases. IC: initial condition, LBL: laminar boundary layer, BC: boundary condition, SD: sinuous disturbances, IST: Isotropic turbulence.

\begin{tabular}{llll}
\hline Case & FST intensity & IC & Inflow BC \\
\hline A & $0 \%$ & Steady LBL profile & LBL + SD \\
B & $6 \%$ & Steady LBL profile & LBL + FST(IST) \\
C & $0.5 \%$ & Case A & LBL + SD + FST(IST) \\
D & $1 \%$ & & \\
\hline
\end{tabular}


separation distance are extracted from the instantaneous flow field. After an appropriate scaling of the turbulence intensities, the resultant velocity perturbations are specified at the inlet boundary at every time step. Details of generating FST are explained in [27].

\section{THE PRESENT METHOD FOR ANALYZING THE DOMINANT LOCAL DYNAMICS OF HAIRPIN VORTICES}

A new method that consists of skeletonization, mathematical term decomposition and the combination of dominant terms around the skeleton points is proposed.

For the skeletonization, a new algorithm for extracting the interior points of vortex structures represented by enclosed iso-surfaces is developed. By this algorithm, vortex structures are converted into point cloud data. Then, nearly mean skeleton points $\mathrm{SP}_{k}\left(k=1, \ldots, N_{s}\right)$ are computed from the point cloud data, and local neighbourhoods $W_{k}\left(k=1, \ldots, N_{s}\right)$ are generated around each of the skeleton points. To identify dominant mathematical terms in each $W_{k}$, the governing field equations are transformed to the form of

$$
\Pi \equiv\left(\frac{\partial \rho}{\partial t}, \rho \frac{\partial u}{\partial t}, \rho \frac{\partial v}{\partial t}, \rho \frac{\partial w}{\partial t}, \frac{\partial T}{\partial t}, \frac{\partial \zeta_{x}}{\partial t}, \frac{\partial \zeta_{y}}{\partial t}, \frac{\partial \zeta_{z}}{\partial t}\right)=R H S .
$$

and the RHS is decomposed into NFD terms. Here, $\rho, u, v, w, T, \zeta_{x}, \zeta_{y}, \zeta_{z}$ are density; the $x, y$, and $z$ components of a velocity vector; and the $x, y$, and $z$ components of a vorticity vector, respectively. NFD terms that have large absolute values (LAVs) in $W_{k}$ are then selected, and finally terms that have strong correlation with RHS in $W_{k}$, which are called strong-correlation (SC) terms, are selected. The combination of the $\mathrm{SC}$ terms and the time-derivative terms of the variables, that is, $\Pi$, constitute a reducedorder model or data-compressed model for the system dynamics within $W_{k}$. When a set of NFD terms, LAV terms and SC terms in $W_{k}$ are denoted as $\Sigma_{N F D, W_{k}}, \Sigma_{L A V, W_{k}}, \Sigma_{S C, W_{k}}$, respectively, the hierarchical relationship $\Sigma_{N F D, W_{k}} \supset \Sigma_{L A V, W_{k}} \supset \Sigma_{S C, W_{k}}$ holds among them.

\subsection{Newly proposed algorithm for judging interior points of a vortex}

The interior region of a vortex tube is expressed using particles extracted by the algorithm devised in this study. This algorithm extracts mesh points surrounded by the iso-surface of the function $f(x, y, z)=$ const. Although an arbitrary function could be used for $f(x, y, z)$, the isosurface of the second invariance of the velocity gradient tensor (SIVGT), which is one of the most prevalent quantities for vortex visualization, is employed in this study. The details of the algorithm are explained in [30].

\subsection{Nearly mean skeleton points}

The present method then computes nearly mean skeleton points of the point clouds obtained as the interior points of vortex structures visualized by SIVGT. The nearly mean skeleton points are computed as the collection of the averaged locations of intersection of rays from interior points with the iso-surfaces, i.e. $\mathrm{PR}_{k, m}$ close to a cut point around the circle $\mathrm{C}$ in figure 1 of [30]. Nearly mean skeleton points are also obtained by the algorithm for the $\mathrm{L}_{1}$-medial skeleton proposed by Huang, et al. [31]. It has been confirmed that both methods give similar results, although they are not shown here. 


\subsection{Local neighbourhood of a skeleton point}

For each skeleton point, the local neighbourhood $W_{k}$ is assumed as mentioned previously. In this study, a rectangular-shaped neighbourhood specified in terms of mesh indices $W_{k}=$ $\left[j_{k}-\Delta, j_{k}+\Delta\right] \times\left[k_{k}-\Delta, k_{k}+\Delta\right] \times\left[l_{k}-\Delta, l_{k}+\Delta\right]$ is generated for a skeleton point for which the closest mesh point is $\left(j_{k}, k_{k}, l_{k}\right)$. Here, $\Delta$ is the half width of $W_{k}$ in the index space. In this study, $\Delta=3$.

\subsection{NFD, LAV, and SC terms}

As mentioned previously, NFD terms are selected in each equation. In Appendix A, the NFD terms that constitute the RHS of Eq. (1) are shown. Although numerous NFD terms appear, not all terms become important. First, LAV terms are evaluated. The magnitude of a NFD term $f_{n}, n=1,2, \ldots$, in $W_{k}$, which is denoted as $\left\|f_{n}\right\|_{W_{k}}$, is defined as the maximum value of the absolute value of $f_{n}$, in $W_{k}$, i.e. $\left\|f_{n}\right\|_{W_{k}} \equiv \max _{(j, k, l) \in W_{k}}\left|f_{n}(j, k, l)\right|$. LAV terms are then defined as NFD terms $f_{n}$ such that $\left\|f_{n}\right\|_{W_{k}} \geq \varepsilon \max _{n}\left\{\left\|f_{n}\right\|_{W_{k}}\right\}$. In this study, $\varepsilon=0.3$.

The LAV terms selected as described above do not necessarily determine the variation of the RHS in $W_{k}$ because these terms may cancel out and only a small subset in the set of LAV terms may control the variation of the RHS. Thus, SC terms that have cross-correlation coefficients with an RHS larger than 0.7 in $W_{k}$ are selected. The reason for selecting the SC terms from the set of LAV terms is to exclude terms of small magnitude that have strong correlation with the RHS. Flow variation in $W_{k}$ is driven by competition between the SC terms. The set of the $\mathrm{SC}$ terms is selected within the neighbourhood $W_{k}$ belonging to a skeleton point $\mathrm{SP}_{k}$, and therefore, the set of SC terms becomes different for different skeleton points.

\section{RESULTS AND DISCUSSION}

\subsection{Effects of FST on hairpin vortex evolution}

Figure 1 shows the vortices generated in the process of the laminar-turbulent transition for Cases A and B. In Case A, symmetric hairpin vortices are generated, and the secondary hairpin vortices are well aligned. The amalgamation of hairpin vortices generated around both sides of the aligned region leads to the formation of turbulent regions. Although not shown here, the skin-friction coefficient $\mathrm{C}_{\mathrm{f}}$ detaches from the laminar correlation at around $x=416 \delta_{\text {in }}$ and reaches the turbulent correlation at around $x=575 \delta_{\text {in }}$ [30]. In Case $\mathrm{B}$, hairpin vortices are much smaller and more asymmetric. Dense and sparse regions are randomly generated. $C_{f}$ detaches from the laminar correlation at around $x=36 \delta_{\text {in }}$ and reaches the turbulent correlation at around $x=81 \delta_{\text {in }}[30]$.

Figure 2 shows the time evolution of hairpin vortices influenced by FST. The hairpin vortices are deformed due to the FST and become asymmetric. Although the arrangement of hairpin vortices is straight in Case A, the arrangement becomes staggered in Cases C and D. Many more secondary hairpin vortices are generated. 


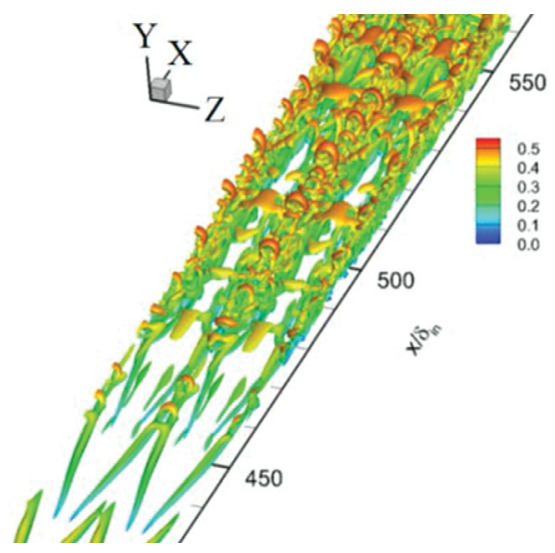

(a) Case $\mathrm{A}$

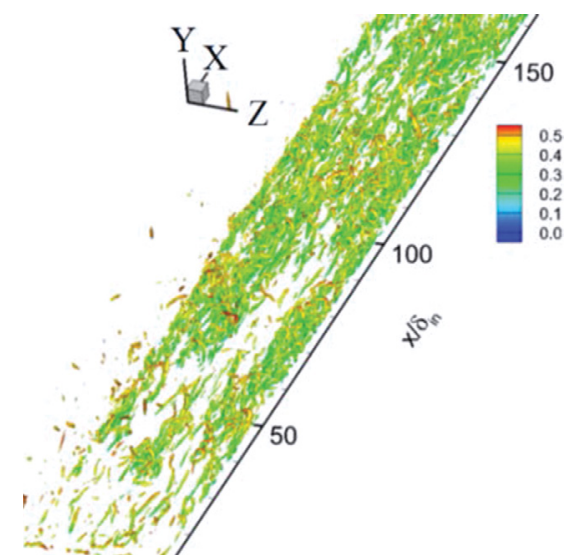

(b) Case B

Figure 1: Instantaneous vortex structures that appear in Cases A and B [28]. Vortex structures are visualized by SIVGT. The colour on the iso-surface and in the legend is the Mach number: (a) Case A and (b) Case B.
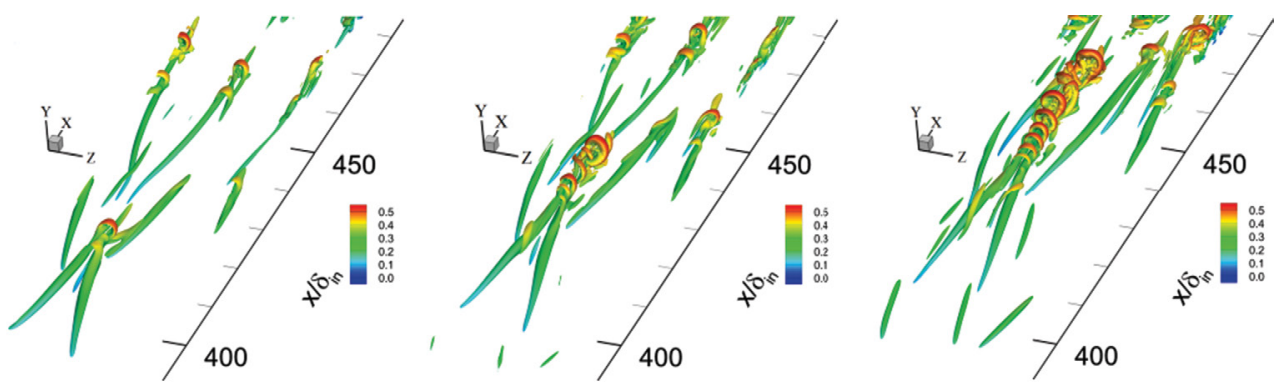

(a) Case $\mathrm{C}$
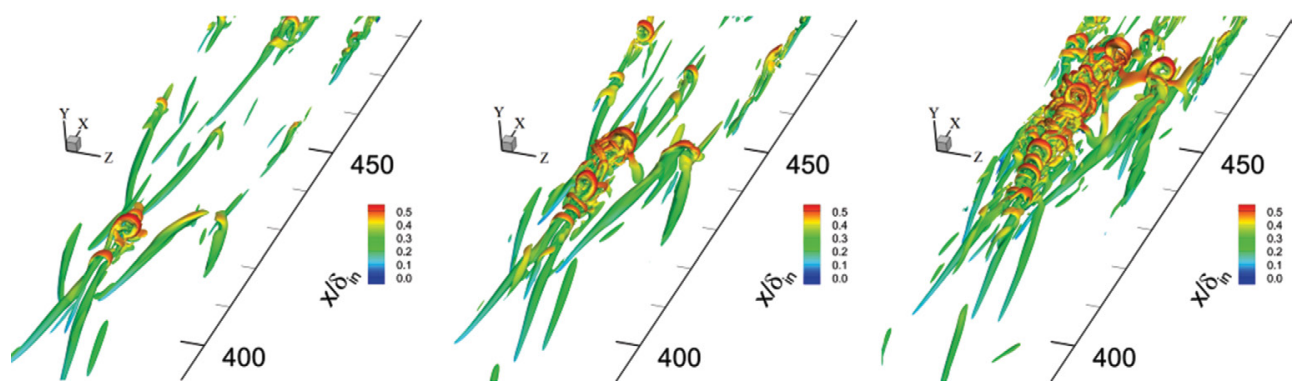

(b) Case D

Figure 2: Effect of FST on the time evolution of hairpin vortices, left: $t=258.0 \delta_{\text {in }} / u_{\infty}$, centre: $t=281.5 \delta_{\text {in }} / u_{\infty}$, right: $t=305.0 \delta_{\text {in }} / u_{\infty}$. The iso-surface quantity and the colour on the iso-surfaces are the same as in Fig. 1 [30]: (a) Case C and (b) Case D. 
5.2 Point cloud representation of vortices, skeletonization and the set of dominant terms

Figure 3 shows the vortex structures represented by the iso-surfaces of SIVGT, and also interior points enclosed by the iso-surfaces. These results show that the present algorithm can successfully pick up interior points surrounded by the SIVGT accurately.

Figure 4 shows the time evolution of hairpin and satellite vortices represented by skeleton points and the iso-surfaces in Case B. For the purpose of visualization, the iso-surfaces are transparent. The skeleton points agree well with the deformation of the iso-surfaces due to the auto-generation of secondary hairpin vortices, and showing that the present skeletonization method can model even small deformations of the iso-surfaces sharply.

LAV terms are extracted for the equations of $\rho, u, v, w, T, \zeta_{x}, \zeta_{y}$, and $\zeta_{z}$ and collected over all skeleton points at $t=0,258.0 \delta_{\text {in }} / u_{\infty}, 281.5 \delta_{\text {in }} / u_{\infty}$, and $305.0 \delta_{\text {in }} / u_{\infty}$. The results are shown in Table 2, where $t=0$ is the initial condition for Case C, in which symmetric hairpin vortices

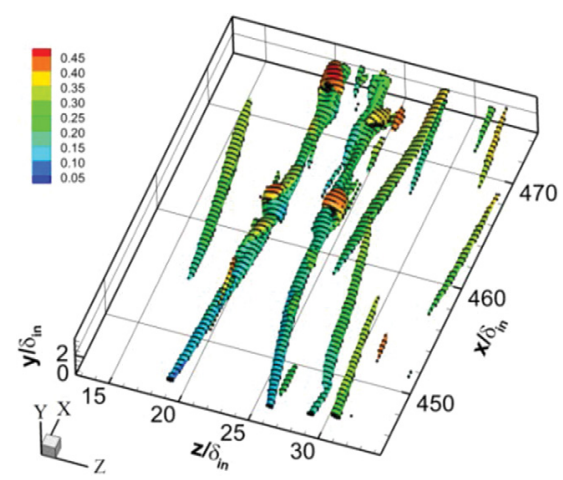

Figure 3: Vortex structures visualized by SIVGT and its interior points (black points) enclosed by the iso-surfaces (Case A). The iso-surface quantity and the colour on the iso-surfaces are the same as in Fig. 1.

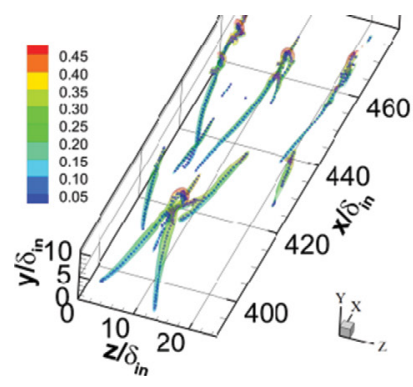

(a) $t=258.0 \delta_{\text {in }} / u_{\infty}$

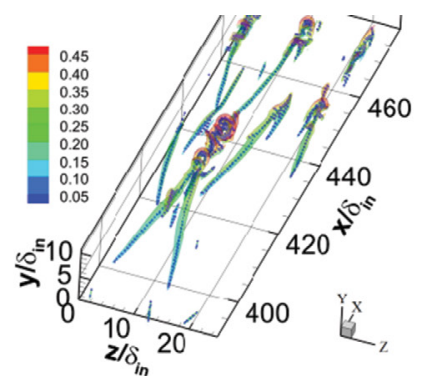

(b) $t=281.5 \delta_{\text {in }} / u_{\infty}$

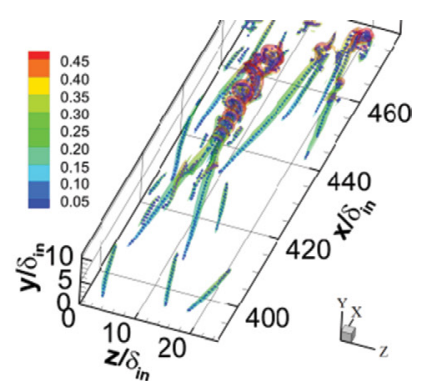

(c) $t=305.0 \delta_{\text {in }} / u_{\infty}$

Figure 4: Time evolution of hairpin and satellite vortices represented by the skeleton points and the transparent iso-surfaces (Case $\mathrm{C}$ ), The iso-surface quantity and the colour on the iso-surfaces are the same as in Fig. 1. (a) $t=258.0 \delta_{\text {in }} / u_{\infty}$, (b) $t=281.5 \delta_{\text {in }} / u_{\infty}$, (c) $t=305.0 \delta_{\text {in }} / u_{\infty}$. 
Table 2: Collection of LAV terms in the equations of $\rho, u, v, w, T, \zeta_{x}, \zeta_{y}$, and $\zeta_{z}$ over all skeleton points at each time, where $t=0$ is the initial condition for Case C.

\begin{tabular}{llllll}
\hline Eq. & & $k, t=0$ & $k, t=258.0 \delta_{\text {in }} / u_{\infty}$ & $k, t=281.5 \delta_{\text {in }} / u_{\infty}$ & $k, t=305.0 \delta_{\text {in }} / u_{\infty}$ \\
\hline$\rho$ & $\left\{r_{k}\right\}$ & $1,3,5$ & $1,3,5$ & $1,3,5$ & $1,3,5$ \\
$u$ & $\left\{u_{k}\right\}$ & $1,2,3,4,5,17$ & $1,2,3,4,5$ & $1,2,3,4,5$ & $1,2,3,4$ \\
$v$ & $\left\{v_{k}\right\}$ & $1,2,3,4,5$ & $1,2,3,4,5$ & $1,2,3,4,5$ & $1,2,3,4,5$ \\
$w$ & $\left\{w_{k}\right\}$ & $1,2,3,4,5,21$ & $1,2,3,4,5$ & $1,2,3,4,5$ & $1,2,3,4,5$ \\
$T$ & $\left\{t_{k}\right\}$ & $10,11,12$ & $10,11,12$ & $10,11,12$ & $10,11,12$ \\
$\zeta_{x}$ & $\left\{o_{k}\right\}$ & $1,2,3,4,5,6,7,8,9,39$ & $1,3,5,6,8$ & $1,2,3,5,6,8,9$ & $1,2,3,4,5,6,8$ \\
$\zeta_{y}$ & $\left\{p_{k}\right\}$ & $1,2,3,4,5,6,7,8,9,44$ & $1,2,3,4,5,6,7,8,9$ & $1,2,3,4,5,6,7,8,9$ & $1,2,3,4,5,6,7,8,9$ \\
$\zeta_{z}$ & $\left\{q_{k}\right\}$ & $1,2,3,4,5,6,7,8,9,82$ & $1,2,3,5,7,8,9,82$ & $1,2,3,5,6,7,8,9,82$ & $1,2,3,4,5,6,7,8,9,82$ \\
\hline
\end{tabular}

are periodically distributed in the flow field. As found from the table, only a small number of NFD terms become large. In the equations of $\rho, u, v, w$, and $T$, the values of the NFD terms are smaller than those for the vorticity equations. The sets of LAV terms do not change significantly for the present range of time spans. However, at $t=0$, the terms of second-order derivatives appear in the sets in contrast to the other times where asymmetric hairpin vortices appear. As found from the iso-surfaces of SIVGT, vortex structures have an inclination towards the wall. Basically, viscous effects become small as vortex structures are lifted up.

Figure 5 shows the skeleton points around which the RHS of the $u$-equation in $W_{k}$ is represented by one SC term, two SC terms, and three SC terms. At all times, the RHS around the skeleton points are mainly determined by one or two SC terms. This property is especially evident around the heads of hairpin vortices where the auto-generation of hairpin vortices takes place.

So far, the present method is applied to the analyses of the instantaneous flow fields. To investigate the variation of the sets of SC terms during vortex evolution, two time series data sets are analysed. Figure 6 shows the time evolution of skeleton points from $t_{1}=258.0 \delta_{\text {in }} / u_{\infty}$ and $t_{2}=281.5 \delta_{\text {in }} / u_{\infty}$. In the figure, the evolution of three selected regions of skeleton points is also shown. Region 1 of $t=258.0 \delta_{\text {in }} / u_{\infty}$ corresponds to the near-head region of a two-leg hairpin vortex, Region 2 corresponds to a leg region, and Region 3 corresponds to the near-head region of a one-leg hairpin vortex. The reason for investigating the evolution of the skeleton points of each region separately is to identify the local dynamics that become strong in each region.

For each region, a corresponding region on the skeleton points at $t=t_{2}$ is found. In each region at $t=t_{1}$, only points corresponding to the skeleton points at $t=t_{2}$ are considered. The corresponding points are found as follows: When the local velocity $\boldsymbol{u}_{i}$ of a skeleton point and time increment $\Delta t=t_{2}-t_{1}$ are taken into account, it is predicted that each point $\boldsymbol{x}_{i}$ at $t=t_{1}$ moves to $\boldsymbol{x}_{i}+\boldsymbol{u}_{i} \Delta t$. By judging the distance between the predicted point and a skeleton point at $t=t_{2}$, if the point $\boldsymbol{x}_{j}$ that satisfies min $\left\|\left(\boldsymbol{x}_{i}+\boldsymbol{u}_{i} \Delta t\right)-\boldsymbol{x}_{j}\right\|<\varepsilon$ is found among the skeleton points at $t=t_{2}$, the point $\boldsymbol{x}_{j}$ is deemed as the corresponding point of $\boldsymbol{x}_{i}$. In this study, $\varepsilon=0.3 \delta_{\text {in }}$ was assumed.

The algorithmically explored results of the transition of the sets of SC terms from $t=t_{1}$ to $t=t_{2}$ associated with Regions 1-3 are shown in Table 3. As found for the $u$-equation, the 

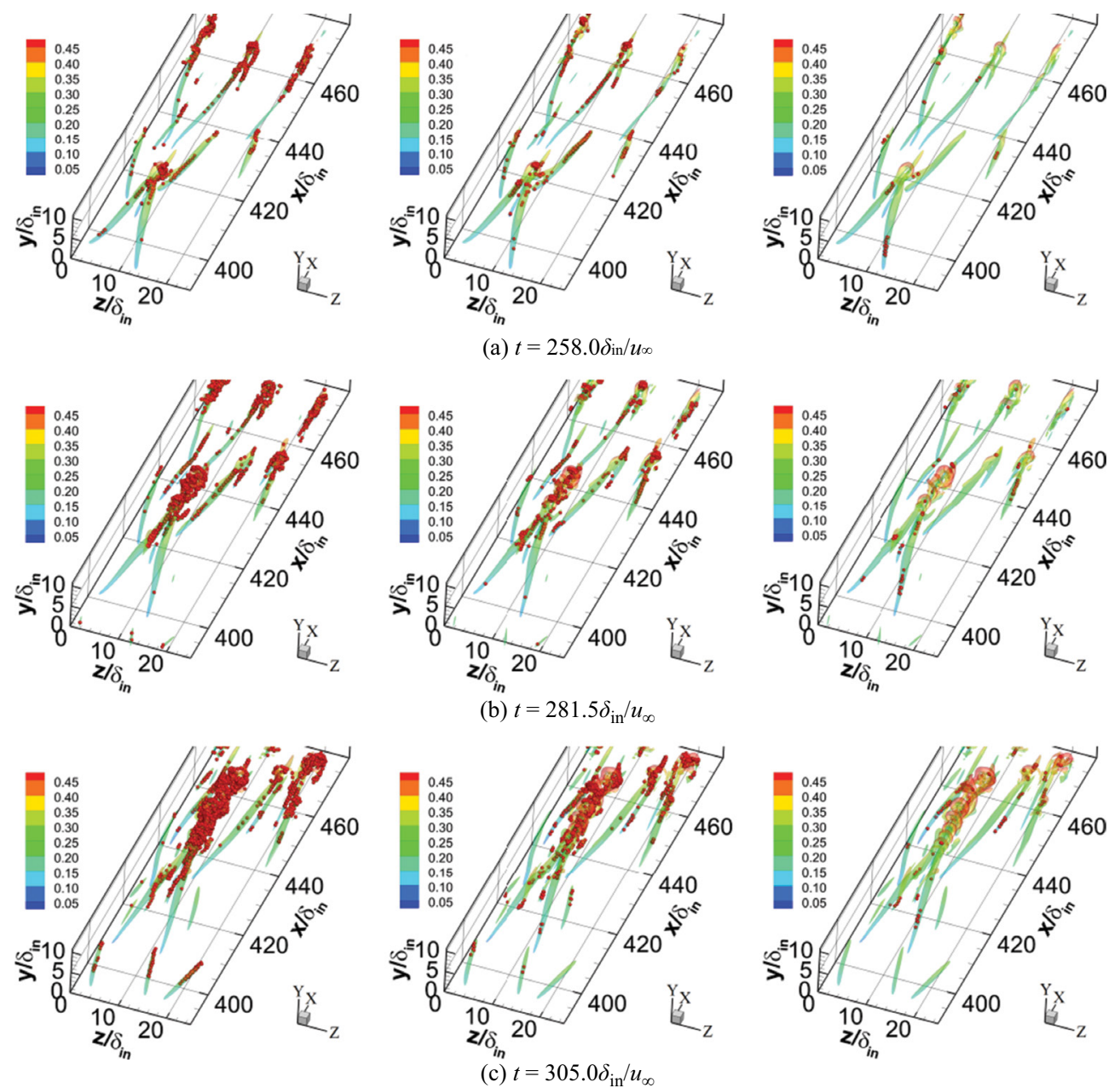

Figure 5: Distribution of skeleton points at which the RHS of the $u$-equation in $W_{k}$ is mainly represented by a single SC term (left), two SC terms (centre), and three SC terms (right) in $W_{k}$ for Case C. The iso-surface quantity and the colour on the iso-surfaces are the same as in Fig. 1. (a) $t=258.0 \delta_{\text {in }} / u_{\infty}$, (b) $t=281.5 \delta_{\text {in }} / u_{\infty}$, (c) $t=305.0 \delta_{\text {in }} / u_{\infty}$.

same transitions $\left\{u_{1}, u_{4}\right\} \rightarrow\left\{u_{1}\right\}$ and $\left\{u_{1}\right\} \rightarrow\left\{u_{1}\right\}$ take place and are most frequently associated with Regions 1 and 3. In contrast, different transitions, such as $\left\{u_{1}, u_{5}\right\} \rightarrow\left\{u_{1}, u_{3}, u_{5}\right\}$ and $\left\{u_{1}, u_{3}, u_{5}\right\} \rightarrow\left\{u_{1}, u_{3}, u_{5}\right\}$ take place in Region 2. In particular, the term $u_{3}$ contains the effects of the $z$ direction, which is reasonable when the fact that hairpin legs are oblique to the $x$ direction is taken into account. The relative frequencies of the second and third transitions are higher in Region 2 compared with Regions 1 and 3. Based on these results, it is found that the regions are categorized inversely from the viewpoint of set transition.

By the present method, the variations of the sets of SC terms that govern the local dynamics are automatically found and categorized during the evolution of hairpin vortices. The amplification and attenuation of disturbance can be evaluated in a Lagrangian manner. These 


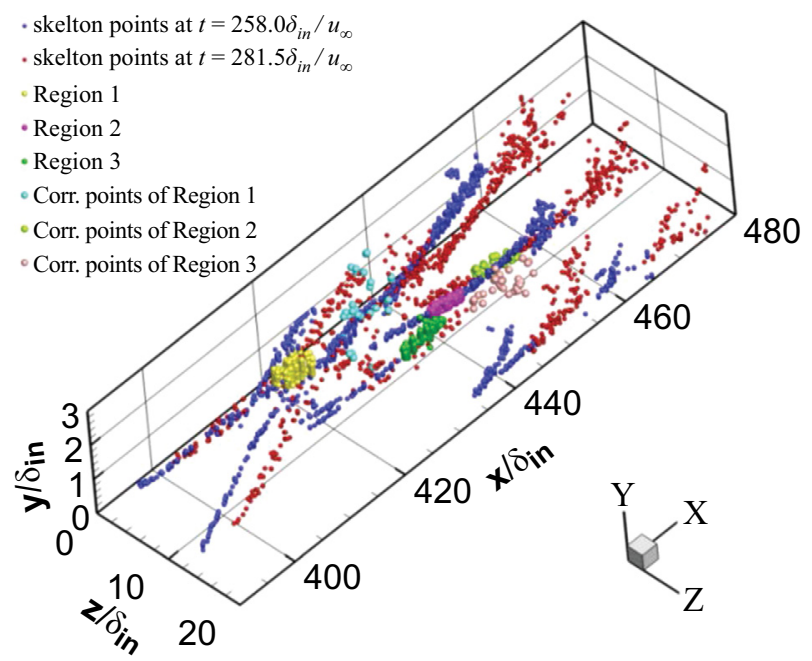

Figure 6: Time evolution of skelton points from $t=258.0 \delta_{\text {in }} / u_{\infty}$ to $t=281.5 \delta_{\text {in }} / u_{\infty}$

Table 3: Transition of the sets of SC terms from $t=t_{1}$ to $t=t_{2}$ associated with Regions 1-3 for $u, v, w, \zeta_{x}$, and $\zeta_{z}$. The number written in parenthesis on the right side of each equation name is the total number of points included in a region that has corresponding points among the skeleton points at $t=281.5 \delta_{\text {in }} / u_{\infty}$. Each column "fq" shows the number of points on which a set transition takes place. The notation $k ; k^{\prime}$ denotes the set transition $\left\{f_{k_{1}}, f_{k_{2}}, \cdots\right\} \rightarrow\left\{f_{k_{1}^{\prime}}, f_{k_{2}^{\prime}}, \cdots\right\}$. The symbol $\varphi$ denotes the null set where no SC terms satisfy the indicated condition. For example, the set transition $\left\{u_{1}, u_{4}\right\} \rightarrow\left\{u_{1}\right\}$ takes place at 15 points out of 40 points associated with Region 1 for the $u$ equation.

\begin{tabular}{|c|c|c|c|c|c|c|c|c|c|}
\hline \multicolumn{10}{|c|}{ Region 1} \\
\hline \multicolumn{2}{|c|}{$u(40)$} & \multicolumn{2}{|c|}{$v(40)$} & \multicolumn{2}{|c|}{$w(40)$} & \multicolumn{2}{|l|}{$\zeta_{x}(40)$} & \multicolumn{2}{|c|}{$\zeta_{z}(40)$} \\
\hline $\mathrm{fq}$ & $k ; k^{\prime}$ & $\mathrm{fq}$ & $k ; k^{\prime}$ & $\mathrm{fq}$ & $k ; k^{\prime}$ & $\mathrm{fq}$ & $k ; k^{\prime}$ & $\mathrm{fq}$ & $k ; k^{\prime}$ \\
\hline 15 & 1,$4 ; 1$ & 21 & $1 ; 1$ & 29 & $1 ; 1$ & 30 & $1 ; 1$ & 3 & $1 ; 1$ \\
\hline 15 & $1 ; 1$ & 4 & 1,$5 ; 1$ & 8 & 1,$2 ; 1$ & 4 & $\varphi ; 1$ & 5 & $1 ; 1,2$ \\
\hline 5 & $\varphi ; 1$ & 4 & $1 ; 1,3$ & 1 & 1,$2 ; 1,2$ & 3 & $1 ; 1,9$ & 2 & $1 ; 1,3$ \\
\hline 3 & $1 ; 1,3,4$ & 4 & 1,$4 ; 1$ & 1 & $1 ; 1,3$ & 2 & $1 ; 1,3$ & 1 & 1,$5 ; 1$ \\
\hline \multicolumn{10}{|c|}{ Region 2} \\
\hline \multicolumn{2}{|c|}{$u(120)$} & \multicolumn{2}{|c|}{$v(120)$} & \multicolumn{2}{|c|}{$w(120)$} & \multicolumn{2}{|c|}{$\zeta_{x}(120)$} & \multicolumn{2}{|c|}{$\zeta_{z}(120)$} \\
\hline 29 & 1,$5 ; 1,3,5$ & 24 & 1,$5 ; 1,3$ & 46 & 1,$4 ; 1,4$ & 34 & 1,$3 ; 1,3$ & 21 & $1 ; 1,5$ \\
\hline 24 & $1,3,5 ; 1,3,5$ & 19 & 1,$5 ; 1,3,5$ & 24 & 1,$4 ; 1,3,4$ & 10 & 1,$8 ; 1,2,8$ & 19 & $1 ; 1$ \\
\hline 14 & 1,$5 ; 1,5$ & 18 & 1,$5 ; 1$ & 20 & $1,3,4 ; 1,3,4$ & 8 & 1,$3 ; 1,2,8$ & 13 & $1,3,82 ; 1,3$ \\
\hline 14 & $1,3,5 ; 1,3$ & 13 & $1,3,5 ; 1,3$ & 19 & $1,3,4 ; 1,4$ & 7 & 1,$8 ; 1,3$ & 12 & 1,$3 ; 1,3,82$ \\
\hline
\end{tabular}


Table 3: (Continued)

\begin{tabular}{|c|c|c|c|c|c|c|c|c|c|}
\hline \multicolumn{10}{|c|}{ Region 3} \\
\hline \multicolumn{2}{|c|}{$u(65)$} & \multicolumn{2}{|c|}{$v(65)$} & \multicolumn{2}{|c|}{$w(65)$} & \multicolumn{2}{|c|}{$\zeta_{x}(65)$} & \multicolumn{2}{|c|}{$\zeta_{z}(65)$} \\
\hline 42 & 1,$4 ; 1$ & 19 & $1,2,4 ; 1$ & 24 & 1,$2 ; 1$ & 41 & $1 ; 1$ & 27 & $1 ; 1$ \\
\hline 10 & $1 ; 1$ & 16 & $1 ; 1$ & 22 & $1 ; 1$ & 8 & $1 ; 1,9$ & 20 & 1,$2 ; 1$ \\
\hline 4 & $\varphi ; 1,4$ & 11 & 1,$4 ; 1$ & 17 & $1 ; 1,4$ & 4 & $1 ; 1,2,8$ & 5 & 1,$9 ; 1$ \\
\hline 3 & 1,$4 ; 1,4$ & 4 & $1,2,4 ; 1,4$ & 2 & $1 ; 1,3$ & 3 & $1 ; \varphi$ & 5 & $1,2,9 ; 1$ \\
\hline
\end{tabular}

aspects give new information on stability when the present method is compared with the conventional linear/nonlinear stability methods based on the Eulerian viewpoint.

\section{CONCLUSIONS}

The effects of FST on the evolution of hairpin vortices during the boundary-layer transition were investigated by DNS. FST intensities of Tu $=0-6 \%$ were considered with a free-stream Mach number of 0.5 . When FST was introduced along with sinuous inlet disturbances, asymmetric hairpin vortices and numerous secondary hairpin vortices, which are qualitatively different from the case of no FST, are generated. The arrangement of hairpin vortices also becomes different. To clarify the dynamics of the hairpin vortices, a new method that evaluates the dominant local dynamics by skeletonization, term decomposition and the combination of dominant terms was proposed. For the skeletonization, a new algorithm for extracting interior points of vortex structures represented by SIVGT was developed. To identify dominant terms, the governing equations were decomposed into NFD terms. In addition, the proposed method was extended to time series flow field data to reveal the variation of the combination of SC terms during the evolution of the vortex structures. The transitions of the sets of SC terms become different according to the vortex region. The present method enables the automatic finding and categorization of the set transition that governs the local dynamics during the evolution of hairpin vortices.

\section{ACKNOWLEDGEMENTS}

The present study was benefited from the outcome of a collaborative research project between Ehime University, Japan, and IHI Corporation. Computations were partly conducted using a supercomputer system at the Japan Aerospace Exploration Agency (JAXA-JSS2). This work was supported by a Grant for Basic Science Research Projects from the Sumitomo Foundation and the Institute of Statistical Mathematics (ISM) Cooperative Research Program 2016 ISM-CRP2019. Computational resources were provided by ISM and JAXA.

\section{APPENDIX A}

Here, NFD terms are derived for the continuity equation, the compressible Navier-Stokes equations, the energy equation, and the vorticity equations [32]. These equations are nondimensional, and the method of non-dimensionalization is same as Matsuura [33].

$\rho, u, v, w, T, \zeta_{x}, \zeta_{y}, \zeta_{z}, \gamma, \mu, M, \operatorname{Re}$ shows density, velocities in the $x, y, z$ directions, temperature, vorticities in the $x, y, z$ directions, the heat ratio, the dynamic viscosity, Mach number and Reynolds number, respectively. $\partial(\cdot ; \cdot)$ denotes a partial differential operator, and $\partial(f ; x)$ denotes $\frac{\partial f}{\partial x}$. The number of letter on the right of the semicolon denotes the order of the derivative, e.g. $\partial(f ; x y z) \equiv \frac{\partial^{3} f}{\partial x \partial y \partial z}$. 
$\partial(\rho ; t)=\sum_{i=1}^{6} r_{i}, r_{1}=-\rho \partial(u ; x), r_{2}=-u \partial(\rho ; x), r_{3}=-\rho \partial(v ; y), r_{4}=-v \partial(\rho ; y), r_{5}=-\rho \partial(w ; z), r_{6}=-w \partial(\rho ; z)$

$\rho \partial(u ; t)=\sum_{i=1}^{21} u_{i}, u_{1}=-\rho u \partial(u ; x), u_{2}=-\rho v \partial(u ; y), u_{3}=-\rho w \partial(u ; z), u_{4}=-T \partial(\rho ; x) /\left(\gamma M^{2}\right), u_{5}=-\rho \partial(T ; x) /\left(\gamma M^{2}\right)$, $u_{6}=-c_{23} \partial\left(\mu^{*} ; x\right) \partial(u ; x), u_{7}=-c_{23} \partial\left(\mu^{*} ; x\right) \partial(v ; y), u_{8}=-c_{23} \partial\left(\mu^{*} ; x\right) \partial(w ; z), u_{9}=-c_{23} \mu^{*} \partial(u ; x x), u_{10}=-c_{23} \mu^{*} \partial(v ; x y)$, $u_{11}=-c_{23} \mu^{*} \partial(w ; x z), u_{12}=2 \partial\left(\mu^{*} ; x\right) \partial(u ; x), u_{13}=2 \mu^{*} \partial(u ; x x), u_{14}=\partial\left(\mu^{*} ; y\right) \partial(v ; x), u_{15}=\partial\left(\mu^{*} ; y\right) \partial(u ; y)$, $u_{16}=\mu^{*} \partial(v ; x y), u_{17}=\mu^{*} \partial(u ; y y), u_{18}=\partial\left(\mu^{*} ; z\right) \partial(u ; z), u_{19}=\partial\left(\mu^{*} ; z\right) \partial(w ; x), u_{20}=\mu^{*} \partial(u ; z z), u_{21}=\mu^{*} \partial(w ; x z)$

$\rho \partial(v ; t)=\sum_{i=1}^{21} v_{i}, v_{1}=-\rho u \partial(v ; x), v_{2}=-\rho v \partial(v ; y), v_{3}=-\rho w \partial(v ; z), v_{4}=-T \partial(\rho ; y) /\left(\gamma M^{2}\right), v_{5}=-\rho \partial(T ; y) /\left(\gamma M^{2}\right)$, $v_{6}=-c_{23} \partial\left(\mu^{*} ; y\right) \partial(u ; x), v_{7}=-c_{23} \partial\left(\mu^{*} ; y\right) \partial(v ; y), v_{8}=-c_{23} \partial\left(\mu^{*} ; y\right) \partial(w ; z), v_{9}=-c_{23} \mu^{*} \partial(u ; x y), v_{10}=-c_{23} \mu^{*} \partial(v ; y y)$, $v_{11}=-c_{23} \mu^{*} \partial(w ; y z), v_{12}=2 \partial\left(\mu^{*} ; y\right) \partial(v ; y), v_{13}=2 \mu^{*} \partial(v ; y y), v_{14}=\partial\left(\mu^{*} ; z\right) \partial(v ; z), v_{15}=\partial\left(\mu^{*} ; z\right) \partial(w ; y)$, $v_{16}=\mu^{*} \partial(v ; z z), v_{17}=\mu^{*} \partial(w ; y z), v_{18}=\partial\left(\mu^{*} ; x\right) \partial(u ; y), v_{19}=\partial\left(\mu^{*} ; x\right) \partial(v ; x), v_{20}=\mu^{*} \partial(u ; x y), v_{21}=\mu^{*} \partial(v ; x x)$ $\rho \partial(w ; t)=\sum_{i=1}^{21} w_{i}, w_{1}=-\rho u \partial(w ; x), w_{2}=-\rho v \partial(w ; y), w_{3}=-\rho w \partial(w ; z), w_{4}=-T \partial(\rho ; z) /\left(\gamma M^{2}\right), w_{5}=-\rho \partial(T ; z) /\left(\gamma M^{2}\right)$, $w_{6}=-c_{23} \partial\left(\mu^{*} ; z\right) \partial(u ; x), w_{7}=-c_{23} \partial\left(\mu^{*} ; z\right) \partial(v ; y), w_{8}=-c_{23} \partial\left(\mu^{*} ; z\right) \partial(w ; z), w_{9}=-c_{23} \mu^{*} \partial(u ; x z), w_{10}=-c_{23} \mu^{*} \partial(v ; y z)$, $w_{11}=-c_{23} \mu^{*} \partial(w ; z z), w_{12}=2 \partial\left(\mu^{*} ; z\right) \partial(w ; z), w_{13}=2 \mu^{*} \partial(w ; z z), w_{14}=\partial\left(\mu^{*} ; x\right) \partial(w ; x), w_{15}=\partial\left(\mu^{*} ; x\right) \partial(u ; z)$, $w_{16}=\mu^{*} \partial(w ; x x), w_{17}=\mu^{*} \partial(u ; x z), w_{18}=\partial\left(\mu^{*} ; y\right) \partial(v ; z), w_{19}=\partial\left(\mu^{*} ; y\right) \partial(w ; y), w_{20}=\mu^{*} \partial(v ; y z), w_{21}=\mu^{*} \partial(w ; y y)$

$\partial(T ; t)=\sum_{i=1}^{30} t_{i}, t_{1}=-u \partial(T ; x), t_{2}=-v \partial(T ; y), t_{3}=-w \partial(T ; z), t_{4}=c_{1} \partial\left(\mu^{*} ; x\right) \partial(T ; x), t_{5}=c_{1} \mu^{*} \partial(T ; x x), t_{6}=c_{1} \partial\left(\mu^{*} ; y\right) \partial(T ; y)$, $t_{7}=c_{1} \mu^{*} \partial(T ; y y), t_{8}=c_{1} \partial\left(\mu^{*} ; z\right) \partial(T ; z), t_{9}=c_{1} \mu^{*} \partial(T ; z z), t_{10}=-(\gamma-1) T \partial(u ; x), t_{11}=-(\gamma-1) T \partial(v ; y), t_{12}=-(\gamma-1) T \partial(w ; z)$, $t_{13}=2 c_{2} \partial(u ; x)^{2}, t_{14}=2 c_{2} \partial(v ; y)^{2}, t_{15}=2 c_{2} \partial(w ; z)^{2}, t_{16}=c_{2} \partial(v ; x)^{2}, t_{17}=c_{2} \partial(u ; y)^{2}, t_{18}=2 c_{2} \partial(u ; y) \partial(v ; x), t_{19}=c_{2} \partial(w ; y)^{2}$, $t_{20}=c_{2} \partial(v ; z)^{2}, t_{21}=2 c_{2} \partial(w ; y) \partial(v ; z), t_{22}=c_{2} \partial(u ; z)^{2}, t_{23}=c_{2} \partial(w ; x)^{2}, t_{24}=2 c_{2} \partial(u ; z) \partial(w ; x), t_{25}=-c_{23} c_{2} \partial(u ; x)^{2}$, $t_{26}=-c_{23} c_{2} \partial(v ; y)^{2}, t_{27}=-c_{23} c_{2} \partial(w ; z)^{2}, t_{28}=-c_{43} c_{2} \partial(u ; x) \partial(v ; y), t_{29}=-c_{43} c_{2} \partial(u ; x) \partial(w ; z), t_{30}=-c_{43} c_{2} \partial(v ; y) \partial(w ; z)$

$\partial\left(\zeta_{x} ; t\right)=\sum_{i=1}^{91} o_{i}, o_{1}=-u \partial\left(\zeta_{x} ; x\right), o_{2}=-v \partial\left(\zeta_{x} ; y\right), o_{3}=-w \partial\left(\zeta_{x} ; z\right), o_{4}=\zeta_{x} \partial(u ; x), o_{5}=\zeta_{y} \partial(u ; y), o_{6}=\zeta_{z} \partial(u ; z)$, $o_{7}=-\zeta_{x} \partial(u ; x), o_{8}=-\zeta_{x} \partial(v ; y), o_{9}=-\zeta_{x} \partial(w ; z), o_{10}=-c_{x 1} \partial(T ; y) \partial(\rho ; z), o_{11}=c_{x 1} \partial(T ; z) \partial(\rho ; y), o_{12}=c_{x 2} \partial\left(\mu^{*} ; x\right) \partial(u ; z)$, $o_{13}=c_{x 2} \partial\left(\mu^{*} ; x\right) \partial(w ; x), o_{14}=c_{x 2} \mu^{*} \partial(u ; x z), o_{15}=c_{x 2} \mu^{*} \partial(w ; x x), o_{16}=c_{x 2} \partial\left(\mu^{*} ; y\right) \partial(v ; z), o_{17}=c_{x 2} \partial\left(\mu^{*} ; y\right) \partial(w ; y)$, $o_{18}=c_{x 2} \mu^{*} \partial(v ; y z), o_{19}=c_{x 2} \mu^{*} \partial(w ; y y), o_{20}=c_{x 2} c_{43} \partial\left(\mu^{*} ; z\right) \partial(w ; z), o_{21}=-c_{x 2} c_{23} \partial\left(\mu^{*} ; z\right) \partial(u ; x), o_{22}=-c_{x 2} c_{23} \partial\left(\mu^{*} ; z\right) \partial(v ; y)$, $o_{23}=c_{43}{ }^{c} x 2^{\mu} \mu^{*} \partial(w ; z z), o_{24}=-c_{23}{ }^{c} x 2^{\mu * \partial(u ; x z), o_{25}=-c_{23}{ }^{c} x 2^{\mu}} \mu^{*} \partial(v ; y z), o_{26}=c_{x 3} \partial\left(\mu^{*} ; x y\right) \partial(u ; z), o_{27}=c_{x 3} \partial\left(\mu^{*} ; x y\right) \partial(w ; x)$, $o_{28}=c_{x 3} \partial\left(\mu^{*} ; x\right) \partial(u ; y z), o_{29}=c_{x 3} \partial\left(\mu^{*} ; x\right) \partial(w ; x y), o_{30}=c_{x 3} \partial\left(\mu^{*} ; y\right) \partial(u ; x z), o_{31}=c_{x 3} \partial\left(\mu^{*} ; y\right) \partial(w ; x x), o_{32}=c_{x 3} \mu^{*} \partial(u ; x y z)$, $o_{33}=c_{x 3} \mu^{*} \partial(w ; x x y), o_{34}=c_{x 3} \partial\left(\mu^{*} ; y y\right) \partial(v ; z), o_{35}=c_{x 3} \partial\left(\mu^{*} ; y y\right) \partial(w ; y), o_{36}=2 c_{x 3} \partial\left(\mu^{*} ; y\right) \partial(v ; y z), o_{37}=2 c_{x 3} \partial\left(\mu^{*} ; y\right) \partial(w ; y y)$,

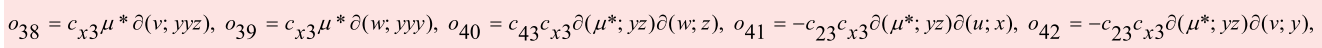

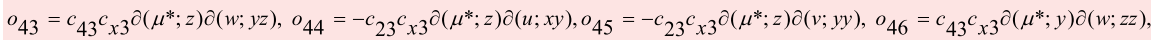

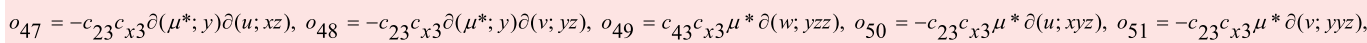
$o_{52}=c_{x 4} \partial\left(\mu^{*} ; x\right) \partial(u ; y), o_{53}=c_{x 4} \partial\left(\mu^{*} ; x\right) \partial(v ; x), o_{54}=c_{x 4} \mu^{*} \partial(u ; x y), o_{55}=c_{x 4} \mu^{*} \partial(v ; x x), o_{56}=c_{43} c_{x 4} \partial\left(\mu^{*} ; y\right) \partial(v ; y)$,

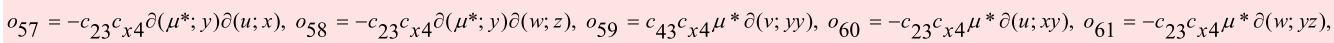
$o_{62}=c_{x 4} \partial\left(\mu^{*} ; z\right) \partial(v ; z), o_{63}=c_{x 4} \partial\left(\mu^{*} ; z\right) \partial(w ; y), o_{64}=c_{x 4} \mu^{*} \partial(v ; z z), o_{65}=c_{x 4} \mu^{*} \partial(w ; y z), o_{66}=c_{x 5^{2}} \partial\left(\mu^{*} ; x z\right) \partial(u ; y), o_{67}=c_{x 5^{2}} \partial\left(\mu^{*} ; x z\right) \partial(v ; x)$, $o_{68}=c_{x 5} \partial\left(\mu^{*} ; x\right) \partial(u ; y z), o_{69}=c_{x 5} \partial\left(\mu^{*} ; x\right) \partial(v ; x z), o_{70}=c_{x 5} \partial\left(\mu^{*} ; z\right) \partial(u ; x y), o_{71}=c_{x 5} \partial\left(\mu^{*} ; z\right) \partial(v ; x x), o_{72}=c_{x 5} \mu^{*} \partial(u ; x y z)$,

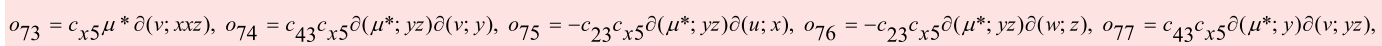

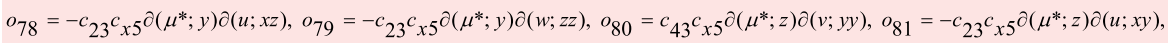
$o_{82}=-c_{23} c_{x 5} \partial\left(\mu^{*} ; z\right) \partial(w ; y z), o_{83}=c_{43^{c} x 5^{\mu}} * \partial(v ; y y z), o_{84}=-c_{23} c_{x 5} \mu^{*} \partial(u ; x y z), o_{85}=-c_{23^{c} x 5^{\mu}} * \partial(w ; y z z), o_{86}=c_{x 5^{2}} \partial\left(\mu^{*} ; z z\right) \partial(v ; z)$, $o_{87}=c_{x 5} \partial\left(\mu^{*} ; z z\right) \partial(w ; y), o_{88}=2 c_{x 5} \partial\left(\mu^{*} ; z\right) \partial(v ; z z), o_{89}=2 c_{x 5} \partial\left(\mu^{*} ; z\right) \partial(w ; y z), o_{90}=c_{x 5} \mu^{*} \partial(v ; z z z), o_{91}=c_{x 5} \mu^{*} \partial(w ; y z z)$ 


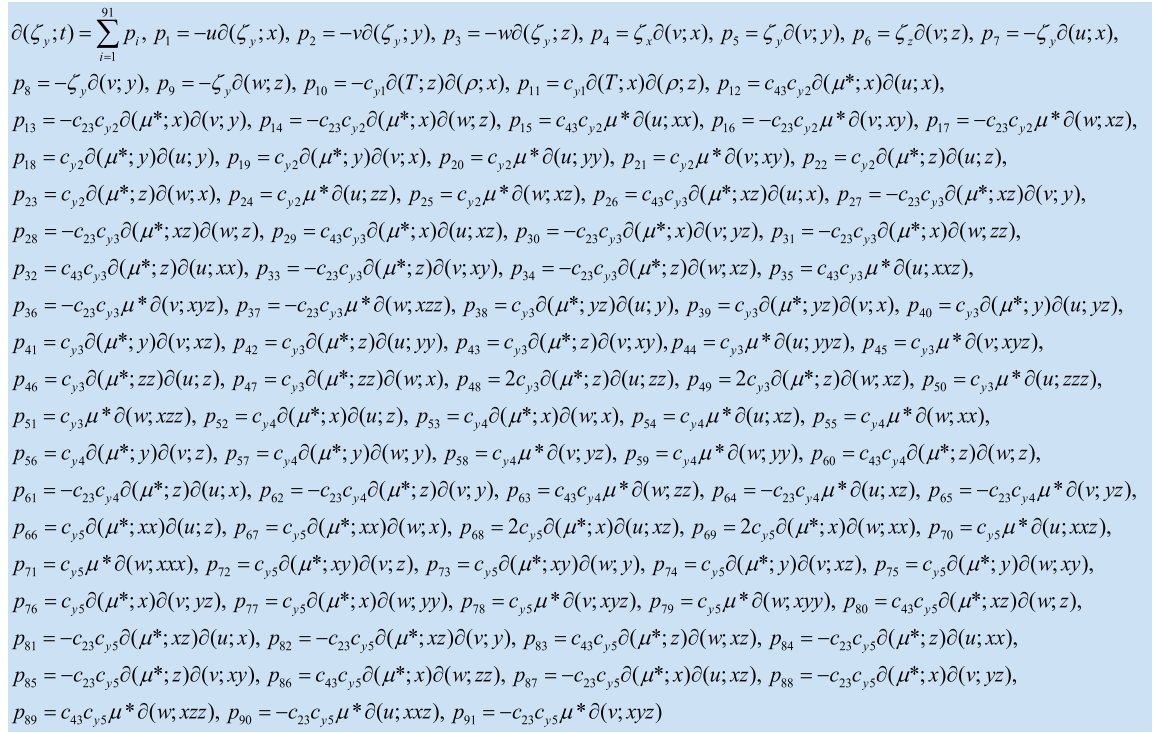

$\partial\left(\zeta_{z} ; t\right)=\sum_{i=1}^{91} q_{i}, q_{1}=-u \partial\left(\zeta_{z} ; x\right), q_{2}=-v \partial\left(\zeta_{z} ; y\right), q_{3}=-w \partial\left(\zeta_{z} ; z\right), q_{4}=\zeta_{x} \partial(w ; x), q_{5}=\zeta_{y} \partial(w ; y), q_{6}=\zeta_{z} \partial(w ; z)$, $q_{7}=-\zeta_{z} \partial(u ; x), q_{8}=-\zeta_{z} \partial(v ; y), q_{9}=-\zeta_{z} \partial(w ; z), q_{10}=-c_{z 1} \partial(T ; x) \partial(\rho ; y), q_{11}=c_{z 1} \partial(T ; y) \partial(\rho ; x), q_{12}=c_{z 2} \partial\left(\mu^{*} ; x\right) \partial(u ; y)$, $q_{13}=c_{z 2} \partial\left(\mu^{*} ; x\right) \partial(v ; x), q_{14}=c_{z 2} \mu^{*} \partial(u ; x y), q_{15}=c_{z 2} \mu^{*} \partial(v ; x x), q_{16}=c_{43} c_{z 2} \partial\left(\mu^{*} ; y\right) \partial(v ; y), q_{17}=-c_{23} c_{z 2} \partial\left(\mu^{*} ; y\right) \partial(u ; x)$, $q_{18}=-c_{23} c_{z 2} \partial\left(\mu^{*} ; y\right) \partial(w ; z), q_{19}=c_{43} c_{z 2} \mu^{*} \partial(v ; y y), q_{20}=-c_{23} c_{z 2} \mu^{*} \partial(u ; x y), q_{21}=-c_{23} c_{z 2} \mu^{*} \partial(w ; y z), q_{22}=c_{z 2} \partial\left(\mu^{*} ; z\right) \partial(v ; z)$, $q_{23}=c_{z 2} \partial\left(\mu^{*} ; z\right) \partial(w ; y), q_{24}=c_{z 2} \mu^{*} \partial(v ; z z), q_{25}=c_{z 2} \mu^{*} \partial(w ; y z), q_{26}=c_{z 3} \partial\left(\mu^{*} ; x x\right) \partial(u ; y), q_{27}=c_{z 3} \partial\left(\mu^{*} ; x x\right) \partial(v ; x)$, $q_{28}=2 c_{z 3} \partial\left(\mu^{*} ; x\right) \partial(u ; x y), q_{29}=2 c_{z 3} \partial\left(\mu^{*} ; x\right) \partial(v ; x x), q_{30}=c_{z 3} \mu^{*} \partial(u ; x x y), q_{31}=c_{z 3} \mu^{*} \partial(v ; x x x), q_{32}=c_{43} c_{z 3} \partial\left(\mu^{*} ; x y\right) \partial(v ; y)$, $q_{33}=-c_{23} c_{z 3} \partial\left(\mu^{*} ; x y\right) \partial(u ; x), q_{34}=-c_{23} c_{z 3} \partial\left(\mu^{*} ; x y\right) \partial(w ; z), q_{35}=c_{43} c_{z 3} \partial\left(\mu^{*} ; y\right) \partial(v ; x y), q_{36}=-c_{23} c_{z 3} \partial\left(\mu^{*} ; y\right) \partial(u ; x x)$, $q_{37}=-c_{23} c_{z 3} \partial\left(\mu^{*} ; y\right) \partial(w ; x z), q_{38}=c_{43} c_{z 3} \partial\left(\mu^{*} ; x\right) \partial(v ; y y), q_{39}=-c_{23} c_{z 3} \partial\left(\mu^{*} ; x\right) \partial(u ; x y), q_{40}=-c_{23} c_{z 3} \partial\left(\mu^{*} ; x\right) \partial(w ; y z)$, $q_{41}=c_{43} c_{z 3} \mu^{*} \partial(v ; x y y), q_{42}=-c_{23} c_{z 3} \mu^{*} \partial(u ; x x y), q_{43}=-c_{23} c_{z 3} \mu^{*} \partial(w ; x y z), q_{44}=c_{z 3} \partial\left(\mu^{*} ; x z\right) \partial(v ; z), q_{45}=c_{z 3} \partial\left(\mu^{*} ; x z\right) \partial(w ; y)$, $q_{46}=c_{z 3} \partial\left(\mu^{*} ; z\right) \partial(v ; x z), q_{47}=c_{z 3} \partial\left(\mu^{*} ; z\right) \partial(w ; x y), q_{48}=c_{z 3} \partial\left(\mu^{*} ; x\right) \partial(v ; z z), q_{49}=c_{z 3} \partial\left(\mu^{*} ; x\right) \partial(w ; y z), q_{50}=c_{z 3} \mu^{*} \partial(v ; x z z)$, $q_{51}=c_{z 3} \mu^{*} \partial(w ; x y z), q_{52}=c_{43} c_{z 4} \partial\left(\mu^{*} ; x\right) \partial(u ; x), q_{53}=-c_{23} c_{z 4} \partial\left(\mu^{*} ; x\right) \partial(v ; y), q_{54}=-c_{23} c_{z 4} \partial\left(\mu^{*} ; x\right) \partial(w ; z), q_{55}=c_{43} c_{z 4} \mu^{*} \partial(u ; x x)$, $q_{56}=-c_{23} c_{z 4} \mu^{*} \partial(v ; x y), q_{57}=-c_{23} c_{z 4} \mu^{*} \partial(w ; x z), q_{58}=c_{z 4} \partial\left(\mu^{*} ; y\right) \partial(u ; y), q_{59}=c_{z 4} \partial\left(\mu^{*} ; y\right) \partial(v ; x), q_{60}=c_{z 4} \mu^{*} \partial(u ; y y)$, $q_{61}=c_{z 4} \mu^{*} \partial(v ; x y), q_{62}=c_{z 4} \partial\left(\mu^{*} ; z\right) \partial(u ; z), q_{63}=c_{z 4} \partial\left(\mu^{*} ; z\right) \partial(w ; x), q_{64}=c_{z 4} \mu^{*} \partial(u ; z z), q_{65}=c_{z 4} \mu^{*} \partial(w ; x z)$, $q_{66}=c_{43} c_{z 5} \partial\left(\mu^{*} ; x y\right) \partial(u ; x), q_{67}=-c_{23} c_{z 5} \partial\left(\mu^{*} ; x y\right) \partial(v ; y), q_{68}=-c_{23} c_{z 5} \partial\left(\mu^{*} ; x y\right) \partial(w ; z), q_{69}=c_{43} c_{25} \partial\left(\mu^{*} ; x\right) \partial(u ; x y)$, $q_{70}=-c_{23} c_{z 5} \partial\left(\mu^{*} ; x\right) \partial(v ; y y), q_{71}=-c_{23} c_{z 5} \partial\left(\mu^{*} ; x\right) \partial(w ; y z), q_{72}=c_{43} c_{25} \partial\left(\mu^{*} ; y\right) \partial(u ; x x), q_{73}=-c_{23} c_{25} \partial\left(\mu^{*} ; y\right) \partial(v ; x y)$, $q_{74}=-c_{23} c_{z 5} \partial\left(\mu^{*} ; y\right) \partial(w ; x z), q_{75}=c_{43} c_{25} \mu^{*} \partial(u ; x x y), q_{76}=-c_{23} c_{z 5} \mu^{*} \partial(v ; x y y), q_{77}=-c_{23} c_{z 5} \mu^{*} \partial(w ; x y z), q_{78}=c_{25} \partial\left(\mu^{*} ; y y\right) \partial(u ; y)$, $q_{79}=c_{25} \partial\left(\mu^{*} ; y y\right) \partial(v ; x), q_{80}=2 c_{25} \partial\left(\mu^{*} ; y\right) \partial(u ; y y), q_{81}=2 c_{25} \partial\left(\mu^{*} ; y\right) \partial(v ; x y), q_{82}=c_{25} \mu^{*} \partial(u ; y y y), q_{83}=c_{z 5} \mu^{*} \partial(v ; x y y)$, $q_{84}=c_{z 5} \partial\left(\mu^{*} ; y z\right) \partial(u ; z), q_{85}=c_{z 5} \partial\left(\mu^{*} ; y z\right) \partial(w ; x), q_{86}=c_{z 5} \partial\left(\mu^{*} ; z\right) \partial(u ; y z), q_{87}=c_{z 5} \partial\left(\mu^{*} ; z\right) \partial(w ; x y), q_{88}=c_{z 5} \partial\left(\mu^{*} ; y\right) \partial(u ; z z)$, $q_{89}=c_{z 5} \partial\left(\mu^{*} ; y\right) \partial(w ; x z), q_{90}=c_{z 5} \mu^{*} \partial(u ; y z z), q_{91}=c_{z 5} \mu^{*} \partial(w ; x y z)$

\section{Here,}

$$
\begin{aligned}
& c_{23}=2 / 3, c_{43}=4 / 3, \mu^{*}=\mu / \operatorname{Re}, c_{1}=\gamma /(\rho \operatorname{Pr}), c_{2}=\gamma(\gamma-1) M^{2} \mu^{*} / \rho \\
& c_{x 1}=1 /\left(\gamma M^{2} \rho\right), c_{x 2}=-\frac{\partial \rho}{\partial y} / \rho^{2}, c_{x 3}=1 / \rho, c_{x 4}=\frac{\partial \rho}{\partial z} / \rho^{2}, c_{x 5}=-c_{x 3}, \\
& c_{y 1}=1 /\left(\gamma M^{2} \rho\right), c_{y 2}=-\frac{\partial \rho}{\partial z} / \rho^{2}, c_{y 3}=1 / \rho, c_{y 4}=\frac{\partial \rho}{\partial x} / \rho^{2}, c_{y 5}=-c_{y 3}, \\
& c_{z 1}=1 /\left(\gamma M^{2} \rho\right), c_{z 2}=-\frac{\partial \rho}{\partial x} / \rho^{2}, c_{z 3}=1 / \rho, c_{z 4}=\frac{\partial \rho}{\partial y} / \rho^{2}, c_{z 5}=-c_{z 3}
\end{aligned}
$$




\section{REFERENCES}

[1] Smith, C.R., Walker, J.D.A., Haidari, A.H. \& Sobrun, U., On the dynamics of near-wall turbulence. Philosophical Transactions of the Royal Society A, 336, pp. 131-175, 1991.

[2] Theodorsen, T., Mechanism of turbulence. Proceeding of Second Midwestern Conference on Fluid Mech., Bull., 149, Ohio State Univ., Columbus, Ohio, 1952.

[3] Lee, C.B. \& Wu, J.Z., Transition in wall-bounded flows. Applied Mechanics Reviews, 61, pp. 030802-1-21, 2008.

[4] Robinson, S.K., Coherent motions in the turbulent boundary layer. Annual Review of Fluid Mechanics, 23(601), pp. 601-639, 1991.

[5] Robinson, S.K., The kinematics of turbulent boundary layer structure. NASA TM 103859, 1991.

[6] Panton, R., Overview of the self-sustaining mechanisms of wall turbulence. Progress in Aerospace Sciences, 37, pp. 341-383, 2001.

[7] Adrian, R.J., Hairpin vortex organization in wall turbulence. Physics of Fluids, 19, pp. 041301, 2007.

[8] Dennis, D.J.C, Coherent structures in wall-bounded turbulence. Annals of the Brazilian Academy of Sciences, 87(2), pp. 1161-1193, 2015.

[9] Moin, P., Leonard, A. \& Kim, J., Evolution of a curved vortex filament into a vortex ring. Physics of Fluids, 29(4), pp. 955-963, 1986. 41.

[10] Hon, T.L. \& Walker, J.D.A, Evolution of hairpin vortices in a shear flow. Computers and Fluids, 20(3), pp. 343-358, 1991.

[11] Singer, B.A. \& Joslin, R.D., Metamorphosis of a hairpin vortex into a young turbulent spot. Physics of Fluids, 6(11), pp. 3724-3736, 1994.

[12] Zhou, J., Adrian, R.J. \& Balachander, S, Autogeneration of near-wall vortical structures in channel flow. Physics of Fluids, 8(1), pp. 288-290, 1996.

[13] Zhou, J., Adrian, R.J. \& Balachandar, S., Mechanisms for generating coherent packets of hairpin vortices in channel flow. Journal of Fluid Mechanics, 387, pp. 353-396, 1999.

[14] Liu, C. \& Chen, L., Parallel DNS for vortex structure of late stages of flow transition. Computers \& Fluids, 45, pp. 129-137, 2011.

[15] Cohen, J., Karp, M. \& Mehta, V., A minimal flow-elements model for the generation of packets of hairpin vortices in shear flows. Journal of Fluid Mechanics, 747, pp. 30-43, 2014.

[16] Eitel-Amor, G., Örlu, R., Schlatter, P. \& Flores, O., Hairpin vortices in turbulent boundary layers. Physics of Fluids. 27, pp. 025108, 2015.

[17] Kim, K., Sung, H. J. \& Adrian, R.J., Effects of background noise on generating coherent packets of hairpin vorticies. Physics of Fluids. 20(105107), pp. 1-10, 2008.

[18] Jiang, M., Machiraju, R. \& Thompson, D., Detection and Visualization of Vortices, The Visualization Handbook, Academic Press, 2005.

[19] Günther, T. \& Theisel, H., The state of the art in vortex extraction, Computer Graphics Forum, 37(6), pp. 149-173, 2018.

[20] Sujudi, D. \& Haimes, R., Identification of swirling flow in 3-D vector fields, AIAA-951715-CP, 1995.

[21] Kida, S. \& Miura, H., Identification and analysis of vortical structures. European Journal of Mechanics - B/Fluids, 17(4), pp. 471-488, 1998.

[22] Peikert, R. \& Roth, M., The "Parallel Vectors" operator - a vector field visualization primitive, Proceedings of IEEE Visualization '99, pp. 263-270, 1999. 
[23] Gelder, A.V. \& Pang, A., Using PVsolve to analyse and locate positions of parallel vectors. IEEE Transactions on Visualization and Computer Graphics, 15(4), pp. 682-695, 2009.

[24] Schafhitzel, T., Vollrath, J.E., Gois, J.P., Weiskopf, D., Castelo, A. \& Ertl, T., Topologypreserving $\lambda_{2}$-based vortex core line detection for flow visualization, Computer Graphics Forum (Eurovis 2008), 27(3), pp. 1023-1030, 2008.

[25] Liu, C., et al., Third generation of vortex identification methods: Omega and Liutex/ Rortex based systems. Journal of Hydrodynamics, 31(2), pp. 205-223, 2019.

[26] Gao, Y., Liu, J., Yu, Y. \& Liu, C., A Liutex based definition and identification of vortex core center lines. Journal of Hydrodynamics, 31(3), pp. 445-454, 2019.

[27] Matsuura, K. \& Kato, C., Large-eddy simulation of compressible transitional flows in a low-pressure turbine cascade. AIAA Journal, 45(2), pp. 442-457, 2007.

[28] Matsuura, K. \& Nakano, M., A throttling mechanism sustaining a hole tone feedback system at very low Mach numbers, Journal of Fluid Mechanics, 710, pp. 569-605, 2012.

[29] Cebechi, T. \& Smith, A.M.O, Analysis of Turbulent Boundary Layer, Academic Press: New York, 1974.

[30] Matsuura, K., DNS investigation into the effect of free-stream turbulence on hairpinvortex evolution, WIT Transactions on Engineering Sciences, 120, pp. 149-159, 2018.

[31] Huang, H., Wu, S., Cohen-Or, D., Gong, M., Zhang, H., Li, G. \& Chen, B., $L_{1}$-medial skeleton of point cloud. ACM Transactions on Graphics, 32(4), pp. 1-8, 2013.

[32] Hirsch, C., Numerical Computation of Internal \& External Flows, Second Edition, Butterworth-Heinemann, 2007.

[33] Matsuura, K., Hairpin vortex generation around a straight vortex tube in a laminar boundary-layer flow, arXiv: 1808.06510v1, pp. 1-41, 2018. 